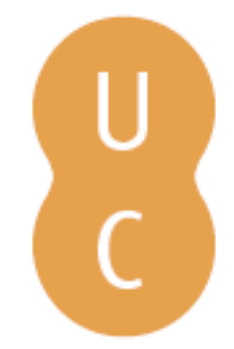

\title{
Rombalina
}

\section{Korrespondenzen mit der Wirklichkeit: Hegel und Deleuze hinsichtlich der Situation des Subjekts in seinen Systemen}

\author{
Autor(es): $\quad$ Rogge, Wibke
}

Publicado por: Imprensa da Universidade de Coimbra

URL

persistente: URI:http://hdl.handle.net/10316.2/31653

DOI: $\quad$ DOI:http://dx.doi.org/10.14195/978-989-26-0205-9_18

Accessed : $\quad$ 26-Apr-2023 12:09:46

A navegação consulta e descarregamento dos títulos inseridos nas Bibliotecas Digitais UC Digitalis, UC Pombalina e UC Impactum, pressupõem a aceitação plena e sem reservas dos Termos e Condições de Uso destas Bibliotecas Digitais, disponíveis em https://digitalis.uc.pt/pt-pt/termos.

Conforme exposto nos referidos Termos e Condições de Uso, o descarregamento de títulos de acesso restrito requer uma licença válida de autorização devendo o utilizador aceder ao(s) documento(s) a partir de um endereço de IP da instituição detentora da supramencionada licença.

Ao utilizador é apenas permitido o descarregamento para uso pessoal, pelo que o emprego do(s) título(s) descarregado(s) para outro fim, designadamente comercial, carece de autorização do respetivo autor ou editor da obra.

Na medida em que todas as obras da UC Digitalis se encontram protegidas pelo Código do Direito de Autor e Direitos Conexos e demais legislação aplicável, toda a cópia, parcial ou total, deste documento, nos casos em que é legalmente admitida, deverá conter ou fazer-se acompanhar por este aviso.

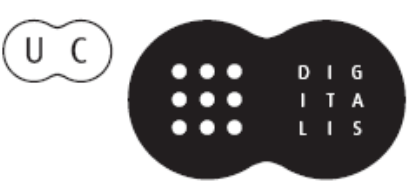


Edmundo Balsemão Pires

Burkhard Nonnenmacher

Stefan Büttner-von Stülpnagel

Editors

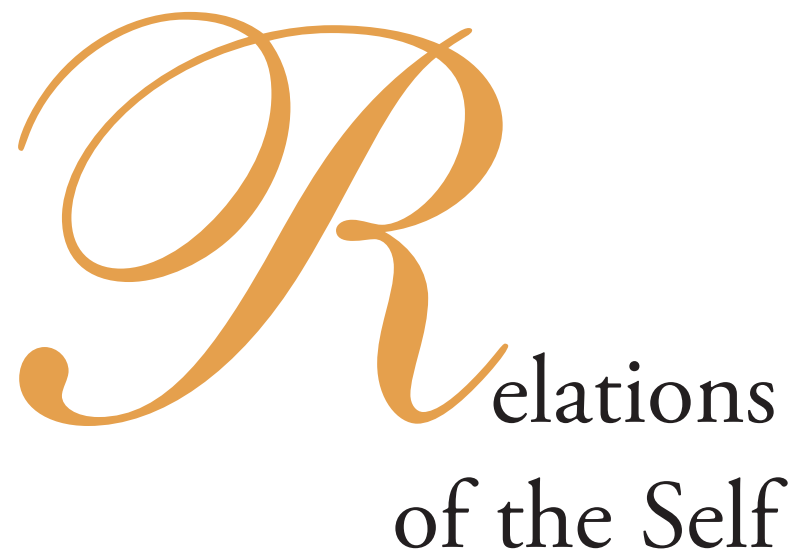




\section{Wibke Rogge \\ Berlin}

\section{KORRESPONDENZEN MIT DER WIRKLICHKEIT. HEGEL UND DELEUZE HINSICHTLICH DER SITUATION DES SUBJEKTS IN SEINEN SYSTEMEN}

Die organische Kausalität des werdenden Selbstbewussteins in Hegels Phänomenologie des Geistes kann auch als eine selbstreferentielle Struktur verstanden werden: Die strategische Teilung des Subjekts in verschiedene gedankliche Figuren führt zu einem Begriff des Selbstbewusstseins, der sich im wesentlichen dadurch auszeichnet, dass ein solches sich auf seine Gegenstände bezieht, indem es sich von ihnen unterscheidet. Schließlich wird damit ein Fremdbezug ermöglicht, für den das Hegelsche Subjekt das geschlossene System des sich entwickelnden Bewusstseins niemals verläßt. Hegel hat seinem Konzept des Geistes eine paradoxe, aber zugleich schlüssige Konstruktion zugrundegelegt, welche die Vorstellung systematischer Identität, als eine „sich-aus-sich“ und „durch-sich“ selbst hervorbringende, befördert. Selbstverhältnis meint bei Hegel immer schon Fremdverhältnis.

Dieser so ansprechenden und eleganten Variante synthetisierenden Denkens möchte ich die eher sperrige Möglichkeit des rhizomatischen Denkens von Gilles Deleuze gegenüberstellen. Deleuze versucht, gerade in Abgrenzung zu Hegel, eine Philosophie des Werdens zu formulieren, die nicht in eine Dialektik mündet, und somit ohne den üblichen Dualismus auskommt. Dabei steht das Erkennen, Produzieren und Bestehen lassen von Differenzen im Vordergrund. Nicht die Dialektik von Herr und Knecht, sondern die interne immanente Differenz bringt Veränderungen hervor. Das implizit Politische liegt in dem „Anti-SubjektKonzept", mit dem er dem autonomen Subjekt der Aufklärung die Differenz und Vielheit entgegensetzt, die sich im Unwahrnehmbar-Werden begrifflich fassen lässt.

Begriffe sind für Deleuze nicht Ideen, im Hegelschen Sinne, die die reale Welt transzendieren und dadurch negieren, sie stehen vielmehr an der Bruchstelle zwischen den Gegenständen, die Veränderung und Durchdringung ermöglicht. Der Begriff stiftet somit neue Verbindungen zwischen den Dingen, indem er ihre virtuellen Unbestimmtheiten verknüpft. Ein solcher Begriff muss in einem positiven Sinne als produktiv verstanden werden. Anders als Hegel kennt Deleuze' Philosophie also keine Negation. Sie trägt die Züge reiner Affirmation der Verknüpfung des bisher Unverbundenen. Deleuze' Philosophie, welche in diesem Zusammenhang auch als Immanenzphilosophie zu verstehen ist, soll die Hegelsche Vorstellung des Subjekts als ein systemimmanentes „perpetuum mobile“ in Frage stellen. 
Dem Problem der Selbstreferentialität hinsichtlich der nie zu entfliehenden subjektiven Perspektive als Erkenntnisquelle des endlichen Bewusstseins begegnen beide Denker auf sehr unterschiedliche Weise. Hegel kann das Subjekt seiner Zeit dem „Fluch der individuellen Identität" durch seine Theorie des vermittelten Selbstbewusstseins als Prinzip seines Systems entheben. Deleuze möchte die Differenz radikaler denken und gerät damit in eine Aporie von Ich und Vor-Ich als Ausgangspunkt der Erkenntnis. Deleuze' Attacke gegen eine prästrukturierte Unantastbarkeit des Denkens macht seine Philosophie zwar interessant für den gegenwärtigen Diskurs, läßt ihn aber methodisch hinter den Hegelschen Begründungsansatz zurückfallen. In Absetzung zu diesem Subjektbegriff kann Hegels Modell neu gelesen und aktualisiert werden. Die Anschlussfähigkeit des spezifisch modernen Diskurses über das "Selbst“ und die theoriegeschichtliche Entwicklung des „Verschwinden des Subjekts“, darf in diesem Zusammenhang nicht aus dem Blick geraten. Denn streitbar ist gerade der Begriff des Subjekts, den Deleuze aufgelöst wissen will, wohingegen Hegel das Subjekt als aufgehoben expliziert hat. Methodisch verfahren beide Denker unterschiedlich haben aber m. E. den gleichen Status von Subjektivität vor Augen. Das Subjekt bringt sich selbst in die Negativität und ist damit seine Negativität, bzw. es beinhaltet seine eigene Negation an-sich. Das Sichselbst-Befragen, das Sich-selbst-in-die-Negativität-Bringen evoziert bei Deleuze eine Aufhebung des Letztbegründungsanspruchs zugunsten einer Genealogie.

Dem Anfang der Wissenschaft der Logik gemäß ist bei Hegel das Sein zunächst das „unbestimmte Unmittelbare" ${ }^{\text {. }}$. Es ist reiner Gedanke und damit Nichts. Sein und Nichts sind unmittelbar identisch. Es gibt, so könnte man sagen, zunächst keine Differenz. Diese aber zeigt sich im Werden und im Entstehen und Vergehen. Im übrigen ist der Begriff des unbestimmt Unmittelbaren eine Abstraktion vom Vermitteltsein alles Seienden, wie das in der Wesenslogik dargetan wird. Insofern muß man das Werden bei Hegel lesen als die Wahrheit des Seins und Nichts, wobei das Sein als aus dem Nichts herkommend verstanden ist und das Nichts aus dem Sein. Ist hier der Umschlag von Sein in Nichts und Nichts in Sein ein quasi unmittelbarer, so ändert sich das schon beim Dasein, wo vom Seienden aus, nämlich der einen Seite von Sein und Nichts die Gegensätzlichkeit von Sein und Nichts ins Spiel kommt. Das Nichts ist dann das je bestimmte Nichts im Verhältnis zu dem je bestimmten Sein als Dasein oder Etwas, von dem es herkommt. Dieser gesamte, man könnte sagen asymmetrische Duktus von Sein und Nichts geht über die Stufen der Seins-, der Wesens- und dann der Begriffslogik. Das Ende der Wesenslogik kulminiert im Begriff der Wirklichkeit, und sie führt über in die Logik des Begriffs. Wirklichkeit, das wird in der Rechtsphilosophie in irritierender Deutlichkeit ausgeführt, ist identisch mit Vernünftigkeit. Nur das Vernünftige ist wirklich und das Wirkliche vernünftig. Vernunft aber ist verknüpft mit der doppelten Gestalt des Begriffs als Begriff des Begriffs, dem Sich-selbst-Begreifen. Dieses Sich-selbst-Begreifen ist vorgestellt als die Bewegung des Begriffs, das Sich-von-sichselbst-Entlassen des Begriffs und das Auf-sich-selbst-Zurückkommen des Begriffs. Es ist, und hierin sind allerdings alle Differenzformen unter dem Gesichtspunkt der Gegensätzlichkeit in die Identität aufgehoben, ein Kreis in Kreisen und repräsentiert in dieser Art eine Selbstgeschlossenheit. Damit generiert die Hegelsche Logik sich aus sich selbst. Sie geht

\footnotetext{
${ }^{1}$ Hegel 1986, 83.
} 
nicht von einem zweifelsfreien Ersten aus, so, wie z. B. Descartes, der über den absoluten Zweifel die absolute Gewißheit im Ich-denke, der res cogitans, gegeben sah. Gerade auch die Hegelsche Logik - wie schon die Phänomenologie des Geistes - ist nicht nur Ausdruck des Zweifels, sondern der absoluten Verzweiflung an jedem festen Halt. Die Dialektik löst alle Positivitäten auf, gelangt aber selbst wieder, so im absoluten Wissen in der Phänomenologie und im Begriff der doppelten Identität in der Logik im Begriff der Idee zu einer zweiten Form von Positivität.

Die Problematik, die sich in der Hegelschen Logik zeigt, ist die Bestimmung der doppelten Negation als Affirmation. Im Affirmativen ist die Dialektik aufgehoben. Das Identische als doppelte Identität, als Identität der Identität und Nichtidentität, setzt die Identität als Aufhebung aller Gegensatzverhältnisse im absoluten Subjekt. Das Subjekt ist identisches Subjekt-Objekt als Subjekt. Beide befinden sich weder im „bloßen Gegensatz zueinander“, noch sind sie identisch im Sinne einer absoluten Subjektivität. Der Rekurs auf ein absolutes Subjekt stellt die Dialektik still. Er ist der geheime Positivismus der Hegelschen Philosophie.

Schon in der Differenzschrift von 1801 umreißt Hegel in Abgrenzung zu Fichte seine Methode anhand der Kategorien Reflexion und Spekulation: Die Reflexion muss, um einen für den Verstand gültigen Satz zu formulieren die Differenz, welche in der Identität des Absoluten und somit auch in der Vernunft vorhanden ist, ausdrücken. „Sie muß, was in der absoluten Identität eins ist, trennen und die Synthese und die Antithese, getrennt in zwei Sätzen, in einem die Identität, im anderen die Entzweiung, ausdrücken. "2 Der Satz A=A, als der Satz der Identität, soll eine Relation aussagen. Er ist aber in seiner Eigenschaft identisch $\mathrm{zu}$ sein der reine Selbstbezug, welcher von allen Differenzen abstrahiert. Die Vernunft gebietet jedoch die Stellen der Relation unterschiedlich zu besetzen. Vernünftigerweise muss man annehmen, dass es neben der absoluten Identität auch eine absolute Differenz geben muss. Damit ist das eine A Subjekt und das andere Objekt. Der Satz ist nun A=B. Nur weil es diesen Satz gibt, kann es auch den ersten Satz, A=A geben. A=B drückt aus, das es eine Relation überhaupt gibt. Das Setzen der Differenz ist also nötig, um einen Begriff von Identität zu erhalten. Die Aussage des zweiten Satzes, A=B ist also der Ausdruck der Vernunft durch den Verstand. Die Beziehung von Vernunft und Verstand umfasst im wesentlichen die Antinomie als Ausdruck der absoluten Identität. A=A kann als die Tätigkeit des reinen abstrakten Denkens verstanden werden und $A=B$ als Nichtdenken. Beide Sätze bedingen sich gegenseitig: „Wenn man bloß auf das Formelle der Spekulation reflektiert und die Synthese des Wissens in analytischer Form festhält, so ist die Antinomie, der sich selbst aufhebende Widerspruch, der höchste formelle Ausdruck des Wissens und der Wahrheit. “3 Um aber ein „System der Realität der Erkenntnis“ zu erhalten, müssen die Sätze als das Ideale (in $\mathrm{A}=\mathrm{A})$ und die Materie $(\mathrm{A}=\mathrm{B})$ verstanden werden. Hegel beweist hiermit, das selbst eine derart schwache Synthese dieser beiden Sätze im Stande ist, die unendliche Fortführung der zirkulären Identität des $\mathrm{A}=\mathrm{A}$ aufzuheben. Es ist wichtig, für ein Grundverständnis Hegelscher Philosophie zu erkennen, dass selbst das dem Denken entgegengesetzte Nicht-Denken letztlich ein Gedachtes ist. Dieser Umstand begründet sich

\footnotetext{
${ }^{2}$ Hegel, 1981, S.39.

${ }^{3}$ Ebd. S.41.
} 
aus seiner Beziehung aufs Denken. Ein reines Nicht-Denken müßte die Beziehung zum Denken überhaupt entbehren, es müßte ihm nicht nur diametral entgegenstehen, sondern vielmehr jenseits des Jenseits also undenkbar sein. Die Entgegensetzung bedeutet also immer schon die Entgegensetzung von Etwas zu sein und damit eine Beziehung auf ein anderes, zumindest das andere seiner selbst, zu haben. Damit ist außerdem gesagt, dass es neben der abstrakten Realität des Denkens noch eine konkrete gibt, welche der „absolute Stoff des Denkens“ ist.

Es ist ihm wichtig zu betonen, dass die rein formale Erscheinung des Absoluten schon die Antinomie in sich birgt. Entgegen einer logisch-zentrierten Philosophie behauptet er den notwendigen Widerspruch in aller Abstraktion: „Einem solchen analytischen Wesen liegt das Bewußtsein nicht zugrunde, daß die rein formale Erscheinung des Absoluten der Widerspruch ist; ein Bewußtsein, das nur entstehen kann, wenn die Spekulation von der Vernunft und dem A=A, als absoluter Identität des Subjekts und Objekts, ausgeht. “"

Ohne die positive Seite des Wissens, die Anschauung ${ }^{5}$, wird die Antinomie im empirischen, bewusstlosen Widerspruch vernichtet. Es soll aber der abstrakte Begriff und das konkrete Sein einer Sache im Wissen vereinigt sein. Es ist also ein transzendentales Wissen von Nöten um die Identität von Subjektivem und Objektivem ins Bewusstsein treten zu lassen. In der transzendentalen Anschauung sind also Sein und Intelligenz vereinigt und je nach Standpunkt, dem der Reflexion oder dem der Notwendigkeit, tritt mehr die Wissens- oder mehr die Seins-Seite hervor. Hiermit ist für Hegel der Ausgangspunkt allen Philosophierens beschrieben. Philosophieren ohne Anschauung ergeht sich in der endlosen oberflächlichen Betrachtung empirischer Entitäten. „In der transzendentalen Anschauung ist alle Entgegensetzung aufgehoben, aller Unterschied der Konstruktion des Universums durch und für die Intelligenz und seiner als ein Objektives angeschauten, unabhängig erscheinenden Organisation vernichtet. Das Produzieren des Bewußtseins dieser Identität ist die Spekulation, und weil Idealität und Realität in ihr eins ist, ist sie Anschauung. "“

In der, gemeinsam mit dem Psychoanalytiker Felix Guattari, verfaßten Schrift „Q'est-ce que la philosophie?" von 1991 veranschlagt Deleuze philosophische Arbeit in erster Linie als Begriffsarbeit. Der Begriff muss dabei als unendlicher dynamischer Prozess verstanden werden, der im Denken der Philosophen stattfindet. Die innere Konsistenz eines Begriffs unterliegt Schwellen- und Werdensprozessen, die untereinander untrennbar miteinander verbunden sind. So ist ein Begriff immer wieder erneut zu hinterfragen, da seine geographischen, historischen, kulturellen, politischen Komponenten sich im stetigen Wandel befinden und insofern den Begriff neu prägen. Durch intensive Neuverknüpfung der Begriffskomponenten wird ein neuer Begriff erschaffen, der die instantane Verfassung des Gedachten aktuell erfasst. Der Begriff wird gewissermaßen aktualisiert. Er wird als Koinzidenz-, Kondensationsoder Akkumulationspunkt seiner eigenen Komponenten angesehen. Er durchwirkt seine Komponenten und ist als Denkakt, als Ereignis zu verstehen: „Der Begriff definiert sich durch die Untrennbarkeit einer unendlichen Zahl von heterogenen Komponenten, die von einem absoluten Überflugspunkt mit unendlicher Geschwindigkeit durchlaufen werden. "7 Er definiert

\footnotetext{
${ }^{4}$ Ebd. S.42.

${ }^{5}$ Es ist an dieser Stelle der veränderte Begriff der Anschauung beim späten Hegel zu beachten. (Anm. W.R.)

${ }^{6}$ Ebd. S.44.

${ }^{7}$ Deleuze/Guattari 1991, S.28.
} 
sich durch seine Konsistenz, er besitzt keine Referenz: Der Begriff ist selbstreferentiell, er setzt sich selbst und setzt seinen Gegenstand gleichzeitig mit seiner Erschaffung.

Ein Begriff ist besser als der vorangehende, wenn er die Probleme seiner Zeit und ihre dazugehörigen Variationen und Resonanzen spürbar macht und ein Ereignis herbeiführt. Die bestehenden Begriffe der Philosophen früherer Zeiten haben demnach ihre Berechtigung allein schon durch ihr Bedürfnis nach Reaktivierung und ihre inspirative Kraft für die Erschaffung neuer Begriffe. Man folgt diesen Philosophen, indem man macht, was sie gemacht haben: Begriffe für Probleme erschaffen, die sich notwendigerweise ändern.

Der Begriff ist somit Selbsterkenntnis: er erkennt das reine Ereignis unabhängig von dem Sachverhalt in dem es sich ereignet. Und damit ist die Hauptaufgabe der Philosophie für ihn beschrieben: Ein Ereignis aus den Dingen freizusetzen, wenn sie Begriffe erschafft.

Da nun deutlich wurde, dass die Begriffe freischwebende, unverbundene Entitäten sind, bedarf es einer Ebene, auf der sich dieselben ansiedeln, in welcher sie sich ein Echo geben. Diese Ebene nennt Deleuze die Immanenzebene. Die Philosophie bietet ein unbegrenztes All-Eines als Schnittfläche für die Begriffe, mit welcher sie in Wechselwirkung stehen. Als Konstruktivismus besitzt die Philosophie zwei komplementäre Aspekte: Begriffe erschaffen und eine Ebene entwerfen. Die Geschwindigkeit des Denkens siedelt sich in diesem Milieu, welches sich unendlich in sich selbst bewegt, an. Deleuze spricht hier vom absoluten Horizont, „der von jedem Beobachter unabhängig ist und das Ereignis als Begriff von jedem Sachverhalt unabhängig macht, in dem es sich verwirklichte. ${ }^{\text {"8 }}$

Jede Philosophie hängt von einer Anschauung ab, welche unaufhörlich entfaltet wird, die Immanenzebene muss also als vorphilosophisch angesehen werden. In ihr verweisen die Begriffe auf ein nicht-begriffliches Verständnis. Dabei existiert dieses „immer-schon“ nicht außerhalb sondern gerade innerhalb der Philosophie. Sie setzt sich dieses als ihre Bedingung voraus. „Das Nicht-Philosophische ist vielleicht tiefer im Zentrum der Philosophie als die Philosophie selbst und bedeutet, daß die Philosophie nicht hinlänglich auf bloß philosophische oder begriffliche Weise verstanden werden kann, sondern in ihrem Wesen sich auch an die Nicht-Philosophen wendet. "9 Die Philosophie impliziert demnach eine Voraussetzung, die sich zugleich von ihr unterscheidet und mit ihr aufs Engste verbunden ist. Ähnlich wie Hegels Anspruch der Spekulation sieht Deleuze das Problem der Philosophie gerade darin, dass sie eine Konsistenz erlangen muss, ohne das Unendliche zu verlieren, in welches das Denken eingebettet ist. Das All-Eine ist der Philosophie nicht entgegengesetzt, sondern ihr immanent. Oder anders: Die Immanenz ist dem Einen immanent. Das Transzendente liegt in der Immanenz selbst.

Das Ereignis ist auf der Immanenzebene vom Subjekt und dessen Individualität abgelöst. Wie eine Art radikaler Empirismus bieten sich auf ihr nur mögliche Welten als Begriffe und Andere als Ausdrücke möglicher Welten dar. „Das Ereignis bezieht nicht das Erleben auf ein transzendentes Subjekt=Ich, sondern bezieht sich im Gegenteil auf den immanenten Überflug eines subjektlosen Feldes; der Andere gibt einem anderen Ich keine Transzendenz zurück, sondern bringt jedes andere Ich auf die Immanenz des überflogenen

\footnotetext{
${ }^{8}$ Ebd. S.43.

${ }^{9}$ Ebd. S.49.
} 
Feldes zurück. "10 Das Subjekt wird damit definiert als nichts weiter, als eine Gewohnheit „ich“ zu sagen. „Es unterscheidet sich von der Erfahrung, sofern es nicht auf ein Objekt verweist und nicht einem Subjekt zugehört (empirische Vorstellung)." ${ }^{11}$ Das transzendentale Feld ist, so Deleuze, ein Prä-reflexiv des Bewußtseins, es ist der Zustand des Ichlosen Bewußtseins. Deleuze postuliert einen „transzendentalen Empirismus” der sich aber fundamental vom rein affektiven unterscheidet. „Mangels Bewußtsein muß sich das transzendentale Feld als eine reine Immanenzebene definieren, da es sich jeder Transzendenz des Subjekts wie des Objekts entzieht." ${ }^{12}$ Das In-sich-sein der Immanenz als Nicht-Subjekt und Nicht-Objekt sichert ihre Absolutheit. Deleuze verweist hier auf Fichte und definiert das transzendentale Feld als ein Leben, das weder von einem Subjekt noch von einem Akt abhängt, sondern als ein „absolutes unmittelbares Bewußtsein, das selbst in seiner Tätigkeit nicht mehr auf ein Sein verweist, sondern sich fortwährend in einem Leben setzt." ${ }^{13}$ Das „transzendentale Feld” wird für Deleuze zu einem Synonym der Identitätslosigkeit und Nichtindividualität. Es wird vielmehr als ein „Netz präindividueller Singularitäten” aufgefaßt, als ein Vor-Nicht-Ich, „Ante-Ich”.

Die Begriffsperson ist nun diejenige, die bei der Erschaffung der Immanenzebene selbst eingreift. Auch sie gehört sowohl in die Ebene hinein, sowie sie von außen auf sie einwirkt. Damit ist eine dynamische Subjektlosigkeit formuliert, die das vormalige Subjekt als Tätigkeit betrachtet: „Ich bin nicht mehr ich, sondern die Fähigkeit des Denkens, sich zu sehen und sich quer durch eine Ebene zu entwickeln, die mich an mehreren Stellen durchquert. " ${ }^{14}$ Die Begriffsperson ist eben in erster Linie ein Werden und damit zwar Subjekt einer Philosophie, aber genau wie diese sich entwickelt und verändert und permanenter Neuschöpfung unterliegt, gilt dies auch für ihr Subjekt. Denken geschieht demnach immer vermittelt durch eine Begriffsperson. Es ist geschieden vom psychosozialen Typen, durch welchen es als Aussage zur Tätigkeit wird. Als Begriffsperson legt sie die Schauplätze des Denkens fest. Sie steht mit ihrem historischen Milieu in Verbindung, welches nur durch psycho-soziale Typen verkörpert werden kann. Der psycho-soziale Typus wandelt individuelle Erlebnisse in Ereignisse des Denkens um und macht sie damit zu Merkmalen einer Begriffsperson. Damit verweisen sie wechselseitig aufeinander, ohne je ineinander aufzugehen.

Begriffsperson und Immanenzebene setzen sich wechselseitig voraus. Die Begriffspersonen bilden die Gesichtspunkte, nach denen sich die Immanenzebenen unterscheiden oder annähern, aber auch die Bedingungen, unter denen jede Ebene von Begriffen derselben Gruppe erfüllt wird. Als unendlicher Akt bringt das Denken endliche Formen hervor. Diese Verschlungenheit bedingt die Vieldeutigkeit der Begriffe, Ebenen und Personen. Die Philosophie begegnet diesem Umstand mit drei Elementen: der prä-philosophischen Ebene, der Immanenzebene; den Pro-philosophischen Personen, die sie erfinden muss und den philosophischen Begriffen, welche es zu erschaffen gilt. Entwerfen, erfinden und erschaffen sind die philosophischen Tätigkeiten nach Deleuze.

\footnotetext{
${ }^{10}$ Ebd. S.56.

${ }^{11}$ Ebd. S.66.

${ }^{12}$ Ebd. S.70.

${ }^{13}$ Ebd. S.72.

${ }^{14}$ Ebd. S.74.
} 
Deleuze attackiert Hegels Konzeption der „Identität der Identität und Nichtidentität” und entlarvt die Differenz dabei als allein auf das Identische reduziert. Die Reduktion der Differenz als bloße Modifikation des Systems, als nur negatives, antithetisches Prinzip, wie es Hegel, so Deleuze postuliert, vermag die Totalität des Systems nicht zu destruieren, noch zu aktualisieren. Der Differenz als bloß antithetisches Moment bleibt letztendlich das versagt, was die absolute Differenz zu leisten vermochte - die absolute Infragestellung der Totalität. Auch bei der noch so geschickt inszenierten Entgegensetzung eines Seins in sein Anderes, die das Sein als Anderssein qualifiziert, bleibt doch das Andere immer im Hegelschen Kreis gefangen und wird durch die positive Aufhebung des dialektischen Prozesses als bloßes Moment der Dialektik seiner alles destruierenden Macht beraubt.

Für Deleuze scheint die Überschreitung der Differenzen weiter zu gehen: Dem Wirklichen kann nicht mehr Realität zukommen als dem Möglichen, da das Wirkliche jeweils in der Vorwegnahme durch diese Vorstellung als Quasi-Übersetzung des Möglichen gedacht ist $^{15}$. Dialektische Wirklichkeitsdeutungen reproduzieren somit eine bestimmte Ordnung oder auch Ideologie - im wahrsten Sinne des Wortes: Neues ist prinzipiell getilgt, da hier Differenz als der Grund von Neuem nach der dialektischen Arbeit nicht mehr existiert, aufgehoben wurde.

Es ist nicht die Denunziation einer bestimmten Philosophie, die Deleuze anstrebt, vielmehr etabliert er eine Strategie, die die Philosophie durch radikale Widersprüche in die Eigentlichkeit der Philosophie zurückbringt. Es geht also darum, etwas Einzigartiges, Bemerkenswertes, Dringendes mit den paradoxen philosophischen Aktivitäten zu entwickeln. Die Kontinuität der philosophischen Reflexion in die Unbeständigkeit der Offenheit hinzuführen, das Zirkuläre einer in sich gefangenen Immanenz zu destruieren, die das Andere, Fremde letztendlich in das Immer-selbe integriert und so die Philosophie zu einer bloßen Bestimmung des immer-schon Bestimmten und Gewußten degradiert, ist die von Deleuze angestrebte Intention. Die sukzessive Integration und Involvierung des Ungedachten als Konstituens der modernen Wirklichkeit defunktionalisiert und entmächtigt die absolute Kognition des „Ich-denke” zugunsten eines permanent sich ereignenden, die Subjektivität in Frage Stellenden Außen. Das „Denken des Außen [...], das in der Selbstgegenwart des Denkens eine irreduzible Alterität und größtmögliche Entfernung zu sich selbst erkennt"16, wird zum Dekonstruktivum des Denkens, das diesen Anstoß nicht denken kann. Das „Denken des Außen” ist die nicht einzunehmende Objektivität, die als absolute Macht mein eigenes Sein permanent relativiert und meine Entwürfe destruiert. „Das AußerPhilosophische wurzelt also tiefer im Inneren der Philosophie als die Philosophie selbst, und was nicht gedacht werden kann, dennoch zum Denken nötigt und einen Taumel ins Cogito eingeführt, ist Immanenz." ${ }^{17}$ Die Anfänglichkeit des Ursprungs der Philosophie ist also faktisch nicht mehr von einem Unbedingten, unbewegten Beweger her zu denken, sondern immer schon durch die Differenz stigmatisiert. Die Philosophie tritt somit aus dem Rahmen metaphysischer Begründungszusammenhänge heraus und fungiert nunmehr als Kritik an

\footnotetext{
${ }^{15}$ http://www.momo-berlin.de/Guenzel_Deleuze.html_ftn 15

${ }^{16}$ Ebd. S.81.

${ }^{17}$ Ebd. S.77.
} 
den Wertsetzungen. So kann denn auch Deleuze dezidiert formulieren: „Die Philosophie läßt sich nicht auf ihre Geschichte reduzieren, weil sich die Philosophie von dieser Geschichte stets losreißt, um neue Begriffe zu erschaffen, die wieder in Geschichte verfallen, aber nicht von ihr herkommen? Ohne die Geschichte bliebe das Werden unbestimmt, bedingungslos, aber das Werden ist nicht geschichtlich." 18

Auf diesen Desubjektivierungs- und Entindividualisierungsprozeß als reinem Destruktionsprozeß, müßte allerdings eine „Re-Konstruktion” folgen. „Man muß auch die Individuationsweisen beschreiben, die es dem präindividuellen transzendentalen Feld erlauben, die Identität empirischer Individuen zu konstituieren." ${ }^{19}$ Diese Rekonstruktion allerdings wird von Deleuze gelöst und endet im Deleuzeschen Desaster, das Ich aus dem Ante-Ich zu konstituieren, da das Ante-Ich auch Ich ist. Selbst die Verlagerung des Ante-Ich in ein Anti-Ich, mithin die absolute Destruktion der Identitäten endet in der Aporie, in der Schizophrenie. „Das Cogito entzieht sich der Alternative von Sinn und Unsinn, Vernunft und Wahnsinn. Es dekonstruiert die strikte Trennung zwischen Parano und Schizo, zwischen Ich und Anti-Ich und zwingt letztlich, den affirmativ-differentiellen Gebrauch der Synthesen - für den gilt: Ich ist ein anderer - zusammenzudenken mit ihrem negativ-identitären Gebrauch, der bewirkt, daß dieser andere immer noch Ich ist." ${ }^{20}$

Die Hauptaufgabe der Philosophie bleibt für Deleuze: Ein Ereignis aus den Dingen freizusetzen, wenn sie Begriffe erschafft. Die Aktualisierung und permanente Infragestellung philosophischer Systeme ist der ansprechende Tenor seiner philosophischen Forderung. Methodisch läßt er sich dennoch auf Winkelzüge ein, wenn er mit der Erschaffung einer Immanenzebene eine produktive Negativität als Anschauung etabliert welche die Philosophie begründen soll. Die Dinge existieren in dem was sie nicht sind, in dem was ihnen entgleitet, so Deleuze, damit kommt er Hegels Anfang in der Wissenschaft der Logik unfreiwillig nah.

Auch die gedankliche Bewegung - weg vom Subjekt - wird von Hegel zugunsten des Subkjekt-Objekts vollzogen. Gerade Hegels, qua Anerkennung realisiertes Selbstbewusstsein bietet eine nicht-identifizierende Subjektstruktur auf, die sich in der Aktualisierung der Lebenswirklichkeit immer wieder aufs neue manifestiert. Letztlich bleibt es dabei: Methodisch ist ein Entweichen aus der subjektiven Perspektive nicht möglich, Selbstreferenz ist im Begründungsanspruch des Subjektbegriffs verankert. Allerdings kann ein ursprünglich durch Fremdbezug vermitteltes selbstbewusstes Subjekt die statische Identitätsstruktur hierarchischer Gesellschaftssysteme in Frage stellen, was einem nomadischen Subjekt des Chaos entgeht.

Nur indem das suchende, sich begründen wollende Ich, sich in diesen Systemen als modernes Ich begreift, als Ich des Scheiterns, als Ich der nachmetaphysischen Wirklichkeit, und sich als kritisches Denken in den Grund des tradierten Denkens denkt, um so das längst Vergangene zu aktualisieren, vermag es die Moderne und sich zu begreifen. Tatsächlich hält doch die wirkliche Gegenwart permanent neue Herausforderungen für das Denken bereit. Denn - und so möchte ich mit einem letzten Zitat von Deleuze schließen: „Die Kraft einer Philosophie wächst nicht mit ihrer logischen Kohärenz oder ihrem begrifflichen Reichtum, sie

\footnotetext{
${ }^{18}$ Ebd. S.92.

${ }^{19}$ Ebd. S.103.

${ }^{20}$ Ebd. S. 110.
} 
bemißt sich einzig daran, ob und in welchem Maße sie noch fähig ist, unserem aktuellen Denken neue Geschwindigkeiten zuzuführen, statt es in der Anschauuung der ewigen Formen stillzustellen. Wichtiger als die Gedanken eines Philosophen ist die Bewegung seines Denkens, die wir als dasjenige erfahren, was uns zu denken gibt, was uns neue Seh- und Hörweisen ebenso wie neue Empfindungsweisen verschafft, indem sie das Denken in eine maximale Entfernung zu dem bringt, was es immer schon gedacht hat, also zu den Mächten des gesunden Menschenverstandes und des Gemeinssinns: sich im Denken fremd zu werden." ${ }^{21}$

${ }^{21}$ Ebd. S.152. 